\section{A generalized computer program for editing narrative text}

\section{JOHN D. MORRIS and LINDA A. MORRIS \\ Georgia Southern College \\ Statesboro, Georgia 30458}

Some attention and effort have been devoted to the computer storage and retrieval of narrative information for purposes ranging from the production of output diagnostic of a subject's score profile on attitudinal or personality measures (Myers, 1962; Guertin, Litcher, Hedges, \& Wilson, 1975) to storing clinical interview material in narrative form (Danskin, Unger, \& Kennedy, 1970). In the former case, the narrative data must be stored in memory and, upon consideration of each component of a subject's score vector, appropriate diagnostic narrative paragraphs can be retrieved and output. This procedure has the advantage of a standardized interpretation by a clinical specialist (usually the test author and/or those consorting with the author). With only the score profile and scale names, the interpreting counselor or the subject may make vast interpretive errors. Accordingly, the purpose of this paper is to describe a computer program which will read in alphameric narrative text and edit it to a specified left- and right-justified line length and also code and punch FORTRAN "DATA" statements containing the edited output. The narrative text could then be compiled by a scoring program and sections would be selected and output diagnostic of a subject's score profile. Alternatively, scoring could be accomplished independently and a purely interpretive program could be built based on these DATA statements.

Program procedure. The narrative text is read from the desired input unit with a desired record length. Right justification is accomplished by inserting blanks into positions selected by the following algorithm setting position priorities. If there is a period in the line with only one blank after it, a blank is first inserted after that period. If no period is found, a blank is inserted after any comma with only one blank following it. In order to facilitate readability and appearance, remaining blanks are inserted between words, beginning from the end of the line, with the most nonblank characters. After extra blanks are inserted between all the words in the line, the "character density" is recalculated and the blank insertion begins from the appropriate end of the line. When the line is right justified, the algorithm stops and edits a new line. In addition, the program provides for a character to signify the premature end of an input record. This is necessary in inserting records or parts

This study was supported in part by a Georgia Southern College Faculty Research Grant. of records into the input file. A paragraph end character is input which signals the algorithm to bypass right justification, ending the paragraph in normal text form. A character can be entered which insures the occurrence of a blank. This is useful in paragraph indentation or centering headings. Also, the user can enter a character signifying a possible syllabication split. This is useful if the text contains large words and is to be output in relatively short lines. Whenever a word appears to be so long that it might cause excessive blanks in right justification, the syllabication character can be entered between syllables. The algorithm will split the word with a hyphen if it appears at the end of an edited line and the split is necessary; otherwise, the syllabication characters are deleted.

FORTRAN DATA statements are coded and punched, manifesting the edited text. These statements are output in a continuous stream of 680 four-character word vectors (constituting the allowable FORTRAN 19 continuation statements). The names of the vectors are an input alphabetic character plus the sequential vector number. Noting where the diagnostic segments of the text begin and end, the user can compile these vectors into a large matrix and select the appropriate segments through the indices of the words.

Input. The user must enter a variable format for reading alphameric text records, the number of records to be edited, the number of characters per record, the line length desired for output (must be a multiple of four), the character the user wishes to specify a prematurely ending input record, the character entered when a blank is specifically desired, the character signifying the end of a paragraph, and the character desired for the alphameric portion of the output DATA statement vector's name. If a blank is entered, no DATA statements will be generated, with the program yielding only the edited printed text.

Output. The left- and right-justified text is printed along with the line numbers and serial number of the four character words contained on each line. It has been found advantageous to iteratively refine the narrative descriptions through a jury method before actually punching the DATA statements. Also of use at this point is the premature end of a record character, allowing the user to change, insert, or delete sections of text without affecting adjacent portions of the text.

After satisfaction with the text has been achieved, the user can produce FORTRAN DATA statements which would be compiled into the narrative text when incorporated into a scoring or interpretive program. The DATA statements are each composed of 680 fourcharacter words coded in a Hollerith format, with the vector name being composed of the desired alpha character and the sequential number of the vector.

Computer and Language. This FORTRAN IV pro- 
gram is in use both on a CDC CYBER 74, in both batch and interactive modes, and on an IBM 370/165.

Availability. The program, with complete documentation and sample input and output illustrating the options described, is available at no cost on request from the senior author at the Department of School Services, Box 8143, Georgia Southern College, Statesboro, Georgia 30458.

\section{REFERENCES}

Danskin. D. G.. Unger, B.. \& Kennedy, C. E. Adapting the computer for narrative material: A progress report. Journal of Counseling Psychology, 1970, 17, 63-66.

Guertin, W. H., Litcher, J. H., Hedges, W. D., \& Wilson, J. T. Multidimensional assessment of philosophy of education. Gainesville, Fl: Parauniversity Resources, 1975.

Myers, I. B. Manual: The Myers-Briggs type indicator. Princeton: Educational Testing Service. 1962. 